\title{
Miniaturization of Flexible Screwing Cell
}

\author{
Asser Vuola, Riku Heikkilä, Timo Prusi, Mikko Remes, Petri Rokka, \\ Niko Siltala, and Reijo Tuokko \\ Tampere University of Technology, Department of Production Engineering \\ Korkeakoulunkatu 6, 33720 Tampere, Finland \\ \{asser.vuola, riku.heikkila, timo.prusi, mikko.remes, \\ petri.rokka, niko.siltala, reijo.tuokko\} atut.fi \\ http://www.tut.fi/tte
}

\begin{abstract}
The research in mini, micro and desktop factories originates from the early 90's and has continued since then by developing the technological basis and different technological building bricks and applications in the field of high- precision manufacturing and assembly of future miniaturised and micro products. This has paved the way for mini, micro and desktop factories which are seen as one potential solution for what kind of production by improving space, energy and material resource utilisation and answering to the needs of design for postponement and customer-close customisation and personalisation. This paper presents one case application for flexible micro factory. Application area is macro world assembly system in miniaturised form. Current trend in this research is the miniaturisation of macro world machines and systems towards more sustainable production technologies.
\end{abstract}

Keywords: Desktop factory, micro factory, parallel robot.

\section{Introduction}

This paper describes the miniaturised concept for flexible screwing cell used in this case for final assembly of a mobile phone. Flexibility has always been one key issue in modern production research. In this application flexibility is defined as follows:

- Work piece fixture and material logistics: No product specific pallets, flat belt conveyor

- Material feeding: Ability to use screws of many sizes easily.

- Machine vision: No fixed sensors or assembly jigs

- Adaptive and easily re-configurable control system

When defining main functionality of assembly device or system, the most important functionality is how to handle and move parts and other materials. Thus we can say that when ever discussed about the assembly system, robot or manipulator must be defined. Typical macro world assembly cell contains a robot or manipulator of some type and its peripherals. When miniaturised assembly systems are designed or defined, all these aspects must be also covered. Miniaturisation of this demonstrator bases on the following aspects [1] [2] [3]: 
- Small, but fast and accurate enough screwing manipulator: In this case a belt driven parallel cartesian manipulator was selected

- Flexible and small feeding device for small parts

- Flexible work piece transportation (flat belt conveyor)

- Integration of the system in $30 \times 20 \mathrm{~cm}$ sized module.

\section{Screwing Manipulator}

Manipulator is the key issue in the assembly system and the most demanding part in the miniaturisation process. Conventional assembly manipulator is typically cartesian, SCARA- or arm-type robot. Common problem for all these types of mechanisms is the scalability of the moving mass relatively to the performance values. Many practical difficulties like cabling, mountings, friction, etc. also occur when one is trying to minimise these structures. Most critical performance deterioration is the decrease of the relative repeatability, caused by the hysteresis in the gear drives and bearings. The use of parallel kinematics in smaller scale robots has been seen as a promising method. Parallel robots are closed loop mechanisms presenting very good performances in terms of accuracy, velocity and rigidity compared to their own size and mass. In fig. 1 and 2 present some typical types of miniature robots using parallel kinematics. [4]

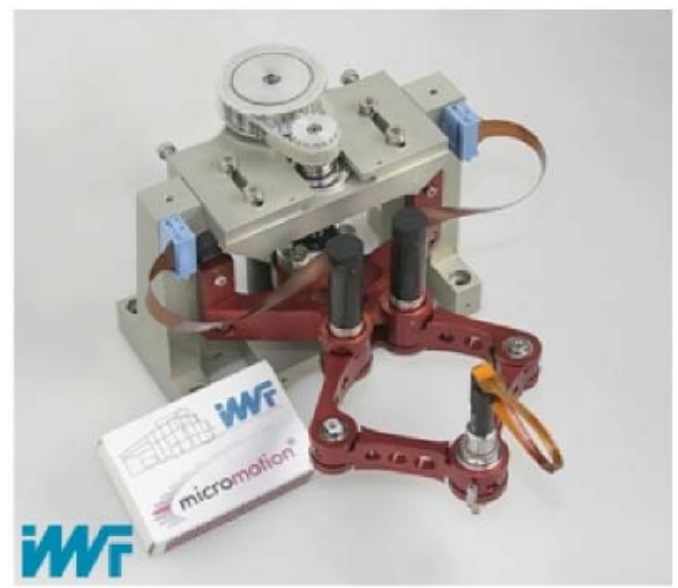

Fig. 1. PARVUS parallel scara-robot [4]

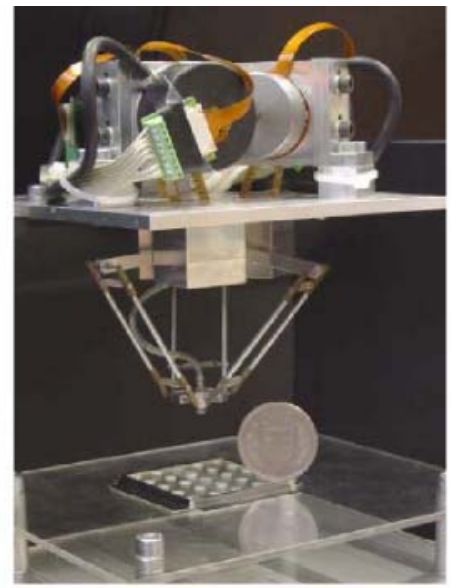

Fig. 2. Pocket delta robot

The main advantage of parallel kinematics in small scale devices is that the electric motor actuators are not a part of the moving mass. The mass of the actuators comes more dominant compared to the mass of the whole device when size goes smaller, especially if sufficient high accuracy is demanded.

So called "H-type" structure has clearly become more common in low-cost but still enough high performance industrial manipulators. Mechanism is belt driven parallel 
structure, where two motors move the same closed loop belt. Thus, motors do not correspond directly to cartesian X-and Y-directions directly. The working principle of the structure is seen in fig. 4. The overall structure of the manipulator is shown in fig. 3. This mechanism was selected for moving the screwing manipulator in XYplane.

Motors M1 and M2 have encoders at the end of the motor shaft. Position is therefore measured only from the driving motors. Indirect feedback and hysteresis caused by the belt drive are the main weaknesses of the structure and thus relatively poor repeatability of positioning was expected. In high precision systems, direct feedback and small hysteresis are the minimum requirements for better accuracy.

Kinematics of the device is quite simple although the motors do not correspond $\mathrm{X}$ and $\mathrm{Y}$ axes directly. $\mathrm{X}$ motion can be derived by moving both motors in the same direction and running the belt through the system. Y motion is respectively derived by running the motors in opposite directions. Idea of the $\mathrm{X}$ - and Y-motions is presented in fig. 4. Command values for driving the motors M1 and M2 are presented in equations 1 and 2. First term in both equations describes $\mathrm{X}$-motion and second term Y-motion.

$$
\begin{aligned}
& M 1=x-y . \\
& M 2=x+y .
\end{aligned}
$$

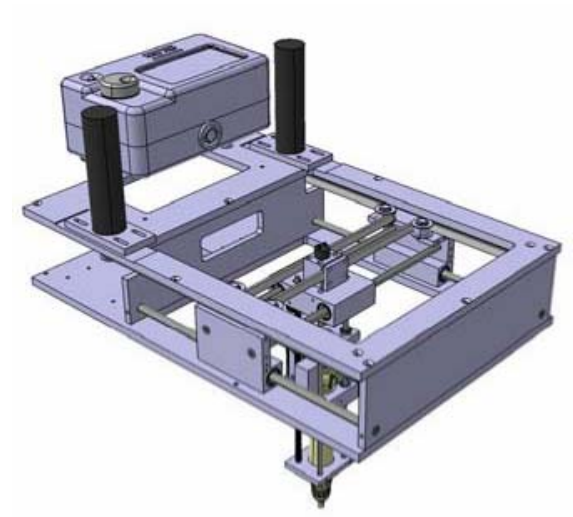

Fig. 3. CAD-model of the H-type structure

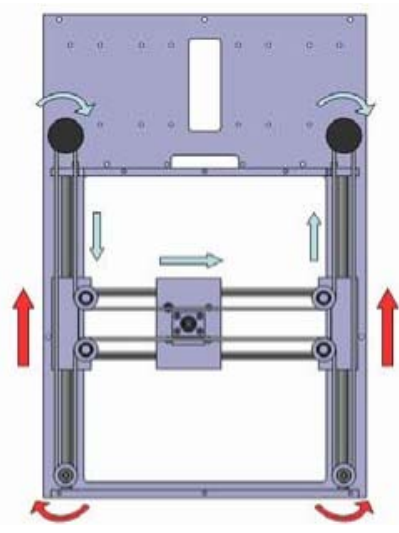

Fig. 4. X- and Y-motions

Z-axis has been implemented with a small ball screw. All guides are linear bushings due to their relative low price and availability compared to the very small prismatic guides. There is also small additional pneumatic extra stroke in Z-direction. With this small cylinder constant pressing force can be achieved for the screwing head by controlling the pressure in the cylinder. Actuator is Festo ADVC-6-10 two actions pneumatic cylinder with $6 \mathrm{~mm}$ piston and $10 \mathrm{~mm}$ stroke.

All motors in the manipulator are Maxon EC-motors, with planetary gearheads, hall sensors and optical encoders. Motion control drives are three small Maxon EPOS 24-1 motion controllers, which are connected together using CANOpen-bus. Position control is made in the embedded PC of the control system. 
Screwing head itself is commercial hand held Atlas Copco MicroTorque ETD M 10 A. It is smallest commercially available electrical screwdriver. Driver has separate control box and it is freely programmable "intelligent" screwdriver. Communication is done with simple IO-handshaking. All process parameters are pre-programmed into the screwing controller. Several different screwing programs can be selected.

\section{Accuracy Measurements}

Repeatability of the positioning of the screwing manipulator was measured according to the ISO 23 0-2 standard [4]. Tests were carried out with Renishaw RLE20 2-axis plane mirror laser interferometer. Test setup is presented in fig. 6. Five target positions and two turning points with $16 \mathrm{~mm}$ steps were used for $\mathrm{X}$ - and $\mathrm{Y}$-axes, covering the whole range of the axes. As described in the standard, each target position must be measured five times in both directions. Positional deviation is calculated and collected for each point. Repeatability is then calculated from the mean positional deviations and standard deviations of the collected data. Repeatability of positioning $\mathrm{R}$ (equation 3) was calculated for both directions, and combined in bidirectional repeatability. The first results of the repeatability tests are shown in table 1.

Table 1. Repeatability of positioning

\begin{tabular}{|l|c|c|c|}
\hline Axis deviation $(\mathrm{mm})$ & Unidirectional $\uparrow$ & Unidirectional & Bidirectional \\
\hline Repeatability of positioning $\mathrm{R}, \mathrm{X}$ & 0,107 & 0,028 & 0,201 \\
\hline Repeatability of positioning $\mathrm{R}, \mathrm{Y}$ & 0,108 & 0,045 & 0,165 \\
\hline
\end{tabular}

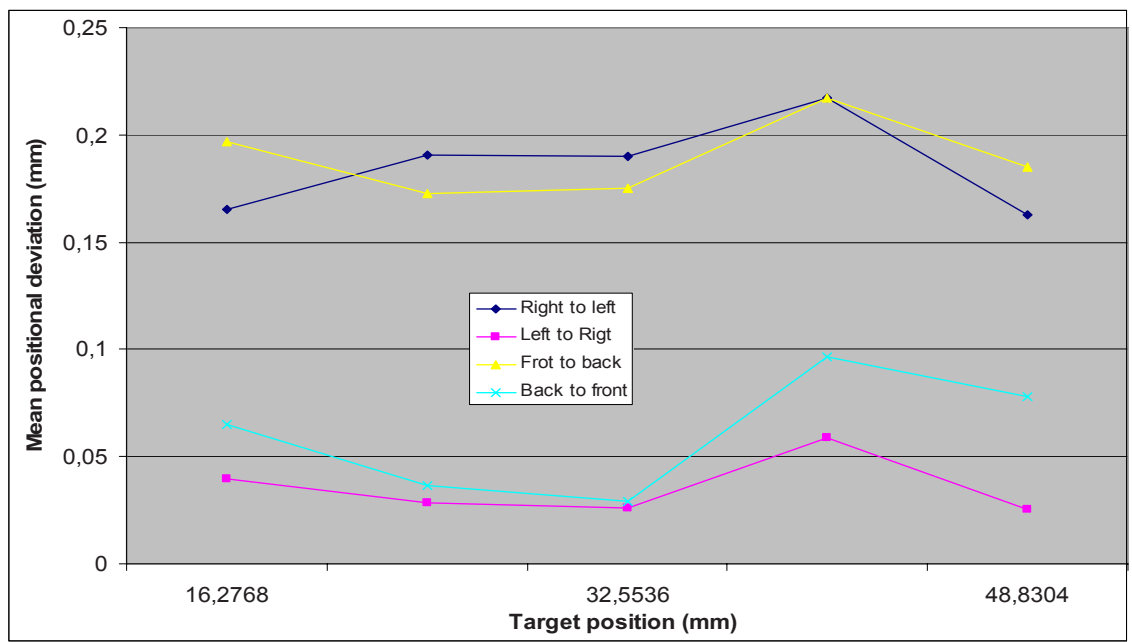

Fig. 5. Mean positional deviations 


$$
R=\max \left[R_{i}\right] .
$$

Where $\mathrm{R}_{\mathrm{i}}$ indicates bidirectional repeatability of positioning at position i [5].

$$
R_{i}=\max \left[2 s_{i} \uparrow+2 s_{i} \downarrow+\left|B_{i}\right| ; R_{i} \uparrow ; R_{i} \downarrow\right] .
$$

Where si indicates the estimator of the unidirectional standard uncertainty of positioning at a position $\mathrm{i}[5]$.

As seen in fig. 5, unidirectional mean positional deviations for both axes are surprisingly good, but there is clearly around $0,15 \mathrm{~mm}$ difference between the approach directions, which clearly indicates the hysteresis of the system.

\section{Machine Vision}

Machine vision is used in two purposes. Firstly to locate screws from the feeder and secondly to recognize work piece features. The Idea behind is that work piece position and orientation is not fixed. Using cameras and machine vision supports the idea of flexibility and easy re-configuration. Vision system is implemented with two small Eye machine vision cameras by IDS. Both cameras have standard C mount- objectives and USB-interface. The two cameras can be seen in fig. 6.

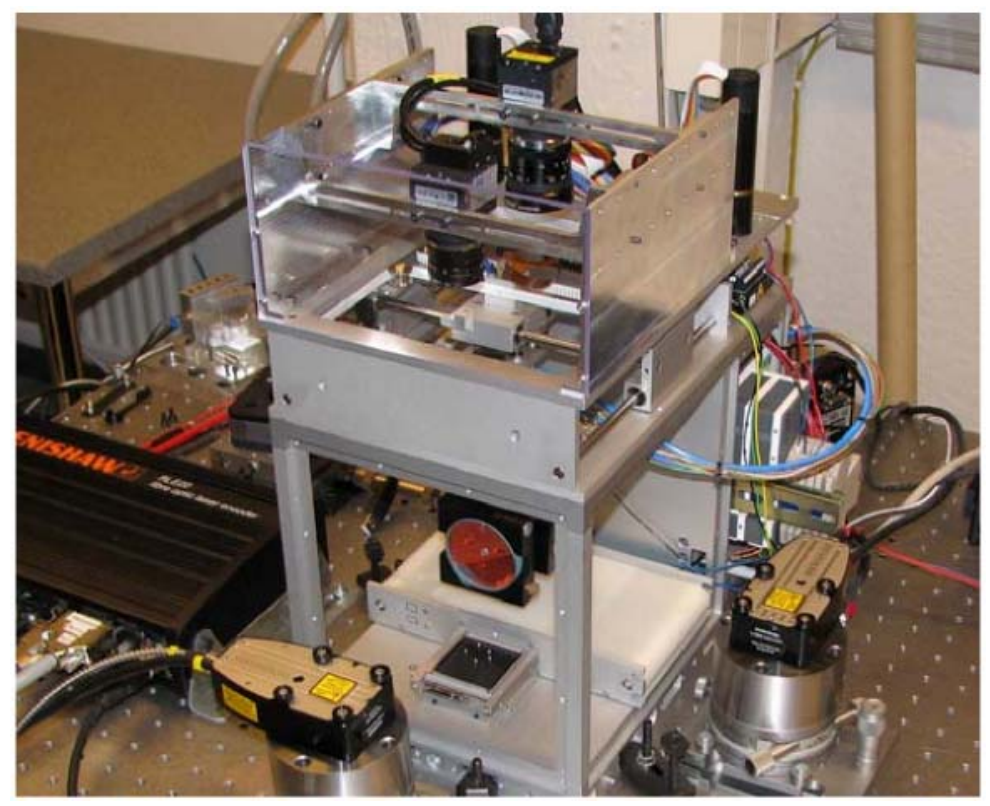

Fig. 6. Test setup and overall structure of the system 
Feeder camera has objective with $12,5 \mathrm{~mm}$ focal length and it gives $100 \mathrm{x} 130$ $\mathrm{mm}$ field of view (FOV). Conveyor camera has objective with $25 \mathrm{~mm}$ focal length and it gives 10x13 mm FOV. Whole working area of the manipulator can thus be covered. Conveyor belt and feeder plate is semi transparent and there is LED backlight inside the structures, but these are not used in this case. But front light is required in both cases.

Phone and screws are found by using simple template matching. From the phone the empty screw holes are a bit harder to find. When phone is black and the holes are black the holes cannot be found without special illumination. Because the holes are hard to find the already screwed holes are detected by using template matching. After the already screwed holes are detected, the places for empty holes are calculated relatively to the phones position and orientation. This is not as accurate as detecting the empty holes but this method does not rely on different illuminations.

\section{Integration and Control}

The presented assembly system has been built to support TUT-Microfatory concept. The main ideas in the concept are modularity and easy re-configurability. Modularity in this case means distributed control architecture and standardised communication and software interfaces. Whole screwing device should be considered as one additional process module with its own control system, which could be assembled to any other base module in the factory. Ethernet is used as higher level communication interface inside the factory concept. Schematic of the control architecture is presented in fig. 7 [6].

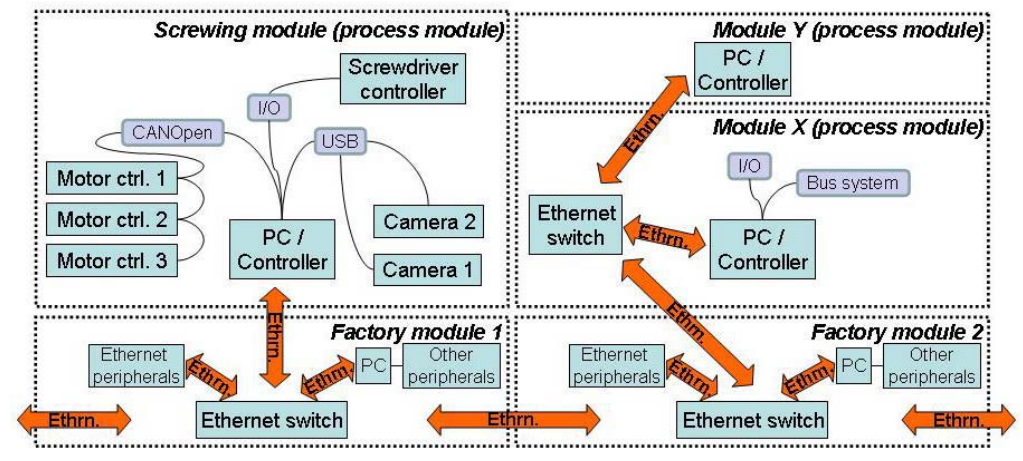

Fig. 7. Control system architecture between the modules

The module or cell internal control structure has more freedom. The used control architecture and bus system can be freely selected by the module provider, until the ethernet is available for communication with other modules. The detailed control architecture of the represented case is shown in Fig 8. A PLC with interpolated numerical control is the master of the whole system. It is programmed through the NC code. The controller is Beckhoff CX1020 with Windows XPe operating system. It 


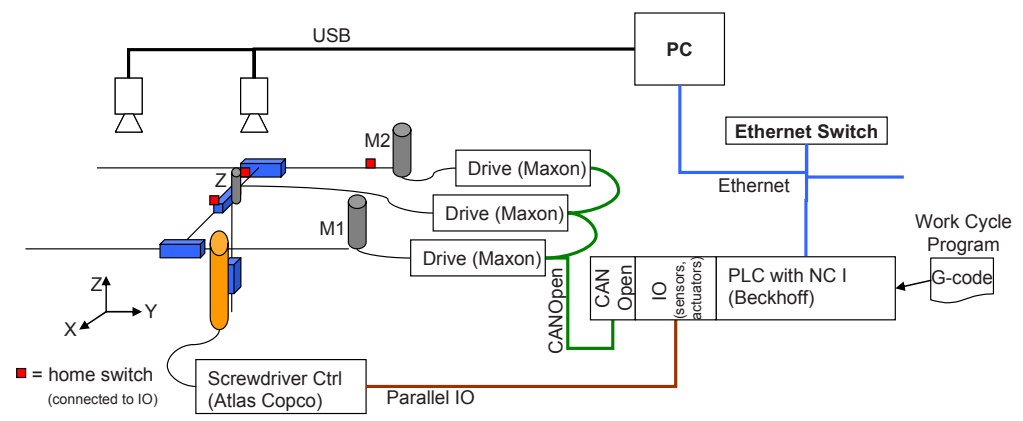

Fig. 8. Control system of the screwing cell

executes the drives for motors (Maxon EPOS 24/1) through CANOpen in velocity mode. Some parallel IOs are connected: Screwdriver controller, sensors like limit switches and actuators like lights or pneumatic valves. The controller communicates with the PC for image capturing and processing actions. PC has two USB cameras which are used to provide information for position and rotation correction before picking and assembly operations.

\section{Conclusions}

In the future the controller for TUT-Factory modules will be changed for smaller, because the industrial components are too large in size to fit into the module. Highly integrated mini PC-board or embedded controller is to be used. This will allow compacted size, optimised feature selection according to the needs, to name some. Current control setup was selected because of stability and reliability of the control, flexibility in IO modules, development time, focus in mechanical solutions and demonstrating the limitations of off-the-self industrial control components within the micro scale production modules.

Complete working cycles was successfully demonstrated after integration and calibration of the system. As described earlier in this paper, compact volume or accuracy were not the main goals for this demonstrator. Miniaturisation of macro world assembly system definitely sets some limits for the performance, but several advantages could be presented in terms of cost efficiency, energy and volume savings.

Certain level of flexibility can be reached by using machine vision as a feedback method for work piece and part localisation and flat belt conveyor for material logistics, instead of product specific pallets.

Complete system test was carried out with a real mobile phone case product containing six M1,6 x 3mm screws. Product is first identified and located with machine vision. Locations of the screws are then calculated in the frame of the manipulator based on a known design data of the case product. Systems then executed a loop until all screw holes are filled. Machine vision is used as feedback and quality 
control for this main loop. After screwing, the complete phone is moved to the next phase with a flat belt conveyor. Performance of the system is presented in table 2 .

Table 2. Preformance of the screwing system

\begin{tabular}{|l|l|}
\hline Overal size & $200 \times 300 \times 400$ \\
\hline Conveyor width & $100 \mathrm{~mm}$ \\
\hline Max. size of the work piece & $100 \times 110 \mathrm{~mm}$ \\
\hline Working area of the robot & $110 \times 135 \times 50$ \\
\hline Max. velocity of the robot & $0,5 \mathrm{~m} / \mathrm{s}$ \\
\hline Repeatability of the robot & $0,2 \mathrm{~mm}$ \\
\hline Possible screw sizes & $\mathrm{M} 1-\mathrm{M} 2,5 \times 6 \mathrm{~mm}$ \\
\hline Max. Number of screw feeders & 3 \\
\hline Average cycle time per screw & $12 \mathrm{~s}$ \\
\hline
\end{tabular}

\section{References}

1. Heikkilä, R., Karjalainen, I., Uusitalo, J., Vuola, A., Tuokko, R.: The concept and first applications of the TUT Microfactory. In: IWMT 2007, 3rd International workshop on microfactory technology, Seogwipo KAL Hotel, Jeju-do, Korea, August 23-24, pp. 57-61 (2007)

2. Heikkilä, R.H., Karjalainen, I.T., Uusitalo, J.J., Vuola, A.S., Tuokko, R.O.: Possibilities of a Microfactory in the Assembly of Small Parts and Products - First Results of the M4-project. In: Proceedings of IEEE International Symposium on Assembly and Manufacturing, ISAM 2007, Ann Arbor, Michigan, USA, July 22-25, pp. 166-171 (2007)

3. Okazaki, Y., Mishima, N., Ashida, K.: Microfactory and Micro Machine Tools. In: Proc. of Korean-Japan Conference on Positioning Technology, Daejeon, Korea (2002)

4. Burisch, A., Wrege, J., Soetebier, S., Raatz, A., Hesselbach, J., Slatter, R.: "Parvus" a Micro-Parallel-Scara Robot for Desktop Assembly Lines. IFIP International Federation for Information Processing, vol. 198 (2006)

5. ISO 230-2 Test code for machine tools - Part 2: Determination of accuracy and repeatability of positioning numerically controlled axes

6. Siltala, N., Heikkilä, R., Tuokko, R.: Architectures and Interfaces for a Micro Factory Concept. In: Ratchev, S., Hauschild, M., (eds.): IPAS 2010, IFIP AICT 315, pp. 293-300 (2010) 